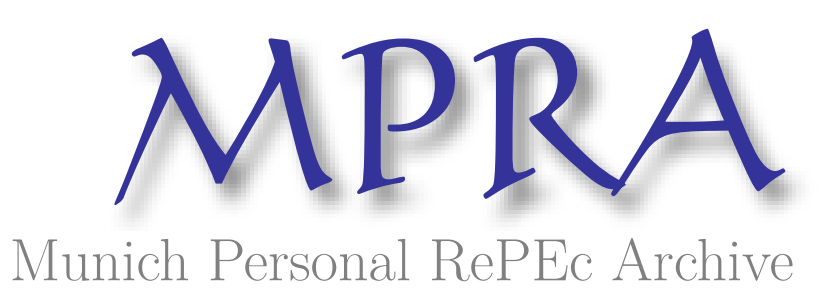

\title{
Extended Ricardian Equivalence Theorem for Helicopter Money
}

Kim, Minseong

2 May 2016

Online at https://mpra.ub.uni-muenchen.de/71067/

MPRA Paper No. 71067, posted 03 May 2016 14:03 UTC 


\title{
Extended Ricardian Equivalence Theorem for Helicopter Money
}

\author{
Minseong Kim
}

$2016 / 05 / 02$

\begin{abstract}
This paper develops the Extended Ricardian Equivalence Theorem for helicopter money. It is shown that helicopter money, or money printing, to finance fiscal spending is inconsistent with existence of an equilibrium under ordinary assumptions used to derive the Ricardian Equivalence theorem. By relaxing some equality constraints into inequality constraints or by an open economy assumption, one may be able to save helicopter money from not being a part of an equilibrium.
\end{abstract}

\section{Extended Ricardian Equivalence Theorem for Helicopter Money}

As price change is assumed to be incorporated rationally into people's decisions, the paper for simplification will assume real variables for every variable. The household faces the following budget constraint:

$$
B_{t+1}+C_{t}+I_{t}=\left(Y_{t}-T_{t}\right)+\left(1+r_{t}\right) B_{t}
$$

where $B_{t}$ is bond holding, $C_{t}$ is consumption, $I_{t}$ is investment, $Y_{T}$ is endowment at time $t$ or output at the aggregate level, $T_{t}$ is taxes, $r_{t}$ is real interest rate. For simplification, the economy is deterministic. Iterating budget constraint forward with present time assumed to be $t=0$,

$$
\lim _{t \rightarrow \infty} \frac{B_{t+1}}{\prod_{u=1}^{t}\left(1+r_{u}\right)}+\sum_{t=0}^{\infty} \frac{C_{t}+I_{t}}{\prod_{u=1}^{t}\left(1+r_{u}\right)}=\sum_{t=0}^{\infty} \frac{Y_{t}-T_{t}}{\prod_{u=1}^{t}\left(1+r_{u}\right)}+\left(1+r_{0}\right) B_{0}
$$

with $\prod_{u=1}^{0}\left(1+r_{u}\right)=1$ for simplifying notation.

Let government budget constraint be:

$$
G_{t}+\left(1+r_{t}\right) D_{t} \leq M_{t}+T_{t}+D_{t+1}
$$


where $M_{t}$ is money obtained by printing money and $D_{t}$ is government debt. Change this into:

$$
G_{t}+\left(1+r_{t}\right) D_{t}+U_{t}=M_{t}+T_{t}+D_{t+1}
$$

where $U_{t}$ refers to unspent money. In the usual equality constraint, $U_{t}=0$, and this will be assumed for initial segments of this paper.

Iterate forward Equation 4:

$$
\sum_{t=0}^{\infty} \frac{G_{t}}{\prod_{u=1}^{t}\left(1+r_{u}\right)}=\sum_{t=0}^{\infty} \frac{T_{t}+M_{t}-U_{t}}{\prod_{u=1}^{t}\left(1+r_{u}\right)}-\left(1+r_{0}\right) D_{0}+\lim _{t \rightarrow \infty} \frac{D_{t+1}}{\prod_{u=1}^{t}\left(1+r_{u}\right)}
$$

Let us substitute Equation 5 into Equation 2 by eliminating $T_{t}$.

$$
\begin{aligned}
\sum_{t=0}^{\infty} \frac{C_{t}+I_{t}}{\prod_{u=1}^{t}\left(1+r_{u}\right)}= & \sum_{t=0}^{\infty} \frac{Y_{t}-G_{t}+M_{t}-U_{t}}{\prod_{u=1}^{t}\left(1+r_{u}\right)}+\left(1+r_{0}\right)\left(B_{0}-D_{0}\right) \\
& +\lim _{t \rightarrow \infty} \frac{D_{t+1}-B_{t+1}}{\prod_{u=1}^{t}\left(1+r_{u}\right)}
\end{aligned}
$$

$1.1 M_{t}=0, U_{t}=0, B_{t}=D_{t}$

This is the ordinary setting for Ricardian equivalence. All bonds are provided by the government - meaning $B_{t}=D_{t}$. But notice that if market clears, then by accounting $C_{t}+I_{t}+G_{t}=Y_{t}$. Thus, all Ricardian equivalence demonstrates is that market clearing is consistent with budget constraints, and for that the choice between money and debt for financing fiscal spending does not matter. It is unclear how the choice affects $Y_{t}$ and $r_{t}$ - more structures are needed than just budget constraints to figure this out. All of these of course assume ordinary assumptions used to derive the Ricardian Equivalence.

$1.2 M_{t} \geq 0, U_{t}=0, B_{t}=D_{t}$

Let us apply market clearing condition $C_{t}+I_{t}+G_{t}=Y_{t}$.

$$
\sum_{t=0}^{\infty} \frac{M_{t}}{\prod_{u=1}^{t}\left(1+r_{u}\right)}=0
$$

But clearly, if $r_{t}>-1$ then if at any $t=s M_{s}>0$ Equation 7 cannot be satisfied. Thus, money printing to finance government spending is inconsistent with existence of equilibrium, unless $r_{s}<-1$ at some time $s$. For the following subsections, $r_{t}>-1$ is assumed.

$1.3 M_{t} \geq 0, U_{t} \geq 0, B_{t}=D_{t}$

In this case,

$$
\sum_{t=0}^{\infty} \frac{M_{t}-U_{t}}{\prod_{u=1}^{t}\left(1+r_{u}\right)}=0
$$


Thus, at some point, if the government does not fully utilize all available money for spending then it is possible that Equation 8 may be satisfied by setting $U_{s}>M_{s}$ at some time $s$, if at time $a<s M_{a}>U_{a}$.

$1.4 M_{t} \geq 0, U_{t}=0, B_{t} \neq D_{t}$

$$
\sum_{t=0}^{\infty} \frac{M_{t}}{\prod_{u=1}^{t}\left(1+r_{u}\right)}=-\left(1+r_{0}\right)\left(B_{0}-D_{0}\right)-\lim _{t \rightarrow \infty} \frac{D_{t+1}-B_{t+1}}{\prod_{u=1}^{t}\left(1+r_{u}\right)}
$$

1.4.1 $\lim _{t \rightarrow \infty} \frac{D_{t+1}-B_{t+1}}{\prod_{u=1}^{t}\left(1+r_{u}\right)}=0$

Then,

$$
\sum_{t=0}^{\infty} \frac{M_{t}}{\prod_{u=1}^{t}\left(1+r_{u}\right)}=-\left(1+r_{0}\right)\left(B_{0}-D_{0}\right)
$$

which suggests that available paths of $M_{t}$ are restricted by states $B_{0}$ and $D_{0}$, which are inherited from the past. If $B_{0}=D_{0}$, then the only path available is $M_{t}=0$.

\subsection{2 $\lim _{t \rightarrow \infty} \frac{D_{t+1}-B_{t+1}}{\prod_{u=1}^{t}\left(1+r_{u}\right)} \neq 0$}

Suppose at some "infinity" limit point $t=s$ (this non-rigorous term is used just for ease of explanation),

$$
\frac{D_{s}-B_{s}}{\prod_{u=1}^{s-1}\left(1+r_{u}\right)}=k \neq 0
$$

Now one also has $1+r_{s}$ term for

$$
\frac{D_{s+1}-B_{s+1}}{\prod_{u=1}^{s}\left(1+r_{u}\right)}=k
$$

Thus,

$$
\lim _{t \rightarrow \infty} \frac{D_{t+1}-B_{t+1}}{D_{t}-B_{t}} \frac{1}{1+r_{t}}=1
$$

Furthermore,

$$
\sum_{t=0}^{\infty} \frac{M_{t}}{\prod_{u=1}^{t}\left(1+r_{u}\right)}=-\left(1+r_{0}\right)\left(B_{0}-D_{0}\right)-k
$$

\subsection{Open economy case}

In an open economy, market clearing implies $Y_{t}=C_{t}+I_{t}+G_{t}+X_{t}-I M_{t}$, where $X_{t}$ is export and $I M_{t}$ is import. Equation 6 then reduces to: 


$$
\begin{aligned}
\sum_{t=0}^{\infty} \frac{I M_{t}-X_{t}}{\prod_{u=1}^{t}\left(1+r_{u}\right)} & =\sum_{t=0}^{\infty} \frac{M_{t}-U_{t}}{\prod_{u=1}^{t}\left(1+r_{u}\right)}+\left(1+r_{0}\right)\left(B_{0}-D_{0}\right) \\
& +\lim _{t \rightarrow \infty} \frac{D_{t+1}-B_{t+1}}{\prod_{u=1}^{t}\left(1+r_{u}\right)}
\end{aligned}
$$

1.5.1 $B_{t}=D_{t}$

Then,

$$
\sum_{t=0}^{\infty} \frac{I M_{t}-X_{t}}{\prod_{u=1}^{t}\left(1+r_{u}\right)}=\sum_{t=0}^{\infty} \frac{M_{t}-U_{t}}{\prod_{u=1}^{t}\left(1+r_{u}\right)}
$$

If every nation prints money to finance government spending at some point, there cannot be any equilibrium. This necessitates $B_{t} \neq D_{t}$ and some policies such as government lending out money $\left(D_{t}<0\right)$ may be needed.

\section{Conclusion}

Again, all the above discussions used standard assumptions used for deriving the Ricardian Equivalence theorem. And the theorem does rely on limits of several variables being finite, as in the standard Ricardian Equivalence theorem. How the result is affected when some of these assumptions are relaxed will be left for future works.

\section{References}

[1] Barro, R. (1974). "Are Government Bonds Net Wealth?" Journal of Political Economy 82 (6): 1095-1117 